\title{
STROKE OUTCOMES IN NON-DIABETIC, DENOVO DIABETIC AND DIABETIC INDIAN PATIENTS MEASURED BY MODIFIED RANKIN SCALE: AN OBSERVATIONAL STUDY
}

\author{
SYED JUNAID AHMED ${ }^{1}$, ABDUR RAHMAN MOHD MASOOD ${ }^{1}$, SAFIYA SUMANA ${ }^{1}$, KHADEER AHMED GHORI ${ }^{1}$, JAVED \\ AKHTAR ANSARI ${ }^{1}$, MUSA KHAN ${ }^{2}$ \\ 1Department of Pharm D, MESCO College of Pharmacy, Hyderabad, TS, India, ${ }^{2}$ Department of General Medicine, Osmania General Hospital, \\ Hyderabad, TS, India \\ Email: javed.ansari47@gmail.com
}

Received: 08 Mar 2017 Revised and Accepted: 19 Jun 2017

\begin{abstract}
Objective: Hyperglycemia is a known risk factor which adversely impacts the outcomes in stroke patients compared to patients with normal blood glucose levels. Patients suffering from an acute stroke who are previously nonhyperglycemic may show elevated blood glucose levels. The present study was designed to measure the outcomes in denovo diabetic and diabetic stroke patients compared to nondiabetics.

Methods: A prospective observational study over a period of $6 \mathrm{mo}$, in which 103 patients were divided into three cohorts based on their blood glucose levels (nondiabetic, denovo diabetic and diabetics). The modified Rankin scale (mRS) score was calculated at in-hospital admission and discharge in these three cohorts. The initial and final scores were correlated and mean differences with respect to outcomes between all the three cohorts was calculated.

Results: The mean mRS at the time of hospital admission in diabetics and nondiabetics was $3.6 \pm 0.81$ and $3.3 \pm 0.78$ which decreased to $2.8 \pm 0.95$ and $2.9 \pm 0.83$ respectively at the time of discharge. The mean mRS score in denovo diabetic stroke patients during in-hospital admission was $4 \pm 0.81$ which was calculated as $3.7 \pm 0.85$ at the time of discharge. The mean difference in mRS score in diabetics vs non-diabetics was found to be $0.73 \pm 0.8$ $(\mathrm{p}=<0.001)$. The mean difference in mRS score of denovo diabetics vs non-diabetics and denovo diabetics vs diabetics was $0.30 \pm 0.63$ and $0.38 \pm 0.61$ respectively $(\mathrm{p}=0.1)$.
\end{abstract}

Conclusion: Results of these observational study in Indian patients, highlights the need for controlling hyperglycemia in stroke patients to improve outcomes and to prevent mortality arising out of acute stroke attacks.

Keywords: Stroke, Hyperglycemia, Denovo diabetes, Modified Rankin scale, Outcomes

(C) 2017 The Authors. Published by Innovare Academic Sciences Pvt Ltd. This is an open access article under the CC BY license (http://creativecommons.org/licenses/by/4.0/) DOI: http://dx.doi.org/10.22159/ijpps.2017v9i8.18378

\section{INTRODUCTION}

Stroke remains the major cause of morbidity and mortality along with being the major cause of adult disability [1]. Stroke is defined as "Rapidly developing clinical signs of focal (or global) disturbance of cerebral function, with symptoms lasting more than $24 \mathrm{~h}$ or leading to death, with no apparent cause other than of vascular origin [2]. Despite the advancements in the diagnosis and pharmacotherapy, stroke remains one of the major neurological diseases often causing gross disability and death [3]. According to WHO, stroke is considered as second leading cause of death above the age of $60 \mathrm{y}$ and fifth leading cause of death between the age group of 15 to $59 \mathrm{y}$ old, it is estimated that 15 million people suffer from stroke annually worldwide, among which 5.5 million die and 5 million are left permanently disabled [4]. The relative number of stroke deaths fell from $35.1 \%$ to $21 \%$, yet approximately $7,95,000$ experience new or recurrent stroke, in 2011 stroke caused 1 of every 20 deaths in USA [5]. Developing countries like India are facing a huge burden of cardiovascular diseases, among which stroke remains one of a leading cause of disability and death. It is estimated that the adjusted prevalence rate of stroke is about 84-262/100,000 in rural and about 334-424/100,000 in urban areas of India. The incidence rate according to recent population-based studies is about 119$145 / 100,000$ [6]. Estimates indicated by Indian Council of Medical Research-ICMR states that among non-communicable diseases (NCD's), stroke contributes to about $41 \%$ deaths and $72 \%$ of Disability Adjusted Life Years (DALY) [7].

Co-morbid risk factors associated with stroke are predictors of poor outcomes in stroke patients [8]. Various risk factors including hypertension, cardiac diseases, diabetes mellitus (DM), smoking, alcohol consumption, stress, and depression could account for $90 \%$ of stroke risk as suggested by an international multicenter study [9]. DM is regarded as an independent risk factor for ischemic stroke, with an estimated prevalence of $21 \%-44.4 \%$ among patients who experience acute ischemic stroke [10]. Several studies have suggested poor functional outcomes for stroke patients with increased blood glucose levels [11-13]. Recent studies have found poor outcomes in prediabetic and denovo diabetes patients in acute ischemic stroke $[14,15]$.

In this study, we aim to compare three cohorts viz diabetic, denovo diagnosed diabetic and nondiabetic patients and correlate blood glucose levels with the outcomes in these patients at the time of hospital admission.

\section{MATERIALS AND METHODS}

We planned an observational study on stroke patients to study the outcomes in relation to blood glucose levels and to determine whether increased glucose levels at hospital admission are associated with poor outcomes.

\section{Study design}

We divided the patients into three cohorts based on the history of blood glucose levels, viz diabetics (cohort 1), denovo diabetics (cohort 2) and nondiabetics (cohort 3). Denovo diagnosed diabetics were differentiated from diabetics based on detailed history, and clinical diagnosis by the attending physician.

It is a hospital-based observational study conducted on 176 stroke patients. Data was collected based on the medical record sheet, patients, and their attendants. The study period was 6 mo. A total of 135 stroke patients were observed according to inclusion and exclusion criteria during the study, 29 patients were lost to follow, and 3 patients were excluded after clinical judgment up leaving 103 patients which were analysed in this study. 
Patients of age $18 \mathrm{y}$ and above of both the genders willing to give their informed consent to participate in the study and patients diagnosed with ischemic and hemorrhagic strokes were included in the present study. However, patients unwilling to give their informed consent, pregnant women, pediatric patients, patients without stroke were excluded from the study.

Outcomes in these patients were evaluated with modified Rankin scale (mRS), which is a measure of global disability and is used as a primary outcome measure for evaluating recovery from stroke. In this study, the poor outcome was defined as Rankin score of 3 or greater. In this study, we have analysed blood glucose levels and mRS outcomes since the time of admission to the day of discharge and correlated it with each cohort.

The study was approved by Institutional Ethics Committee (No. $\mathrm{MCP} / \mathrm{PD} / \mathrm{PR} / 05$ ) before the commencement of the project. Informed consent was taken from patients. Data collection form was designed covering all necessary parameters for the conduct of the study. The blood glucose level was recorded on daily basis and mRS scoring at the time of admission and on the day of discharge was done. Blood glucose levels were correlated the with the mRS score in these patients. Follow-up of patients was done.

The study was performed in inpatient Department of General Medicine of Osmania General Hospital, a tertiary care teaching hospital in Hyderabad, Telangana state, India, during the period January 2015 to June 2015. It is a 1500-bedded government setup teaching hospital providing specialised and super specialized health care services to all the people.

\section{Statistical analysis}

One sample t-test was used to compare the means and to determine if the difference existed between cohorts of patients. For all analyses, the level of significance was set at $p<0.05$. Descriptive statistics was used and data were presented as mean difference and+/-standard deviation (SD). Statistical analysis was performed using the SPSS for Windows, version 20.0 (IBM Inc.)

\section{RESULTS}

A total of 103 patients were assessed for their blood glucose levels and mRS scores during their stay at the hospital for six months. These patients were divided into three cohorts with respect to their blood glucose levels at the time of hospital admission viz diabetics (cohort 1), denovo diabetic (cohort 2) and nondiabetic (cohort 3). Blood glucose levels were recorded on each day, mRS scores at the time of admission and discharge.

The mean age of stroke in diabetic patients was $57.8 \pm 11.13$ (median = 60 , range $=40-85)$ years, 56.69 $\pm 15.71($ median $=60$, range $=30-85)$ years in denovo diabetic patients and $52.54 \pm 14.64$ (median $=51$, range $=22-90$ ) years in nondiabetic patients. There was male dominance in our study with $32(71 \%)$ being diabetic males compared to $13(29 \%)$ diabetic females, there were nine male patients (69\%) compared to four female patients (31\%) in denovo diagnosed diabetic group. Twenty-five (56\%) males were nondiabetic compared to 20 (44\%) females. Hypertension as a co-morbid factor was most prevalent in the diabetic group of 38 patients $(84 \%)$ presenting with high blood pressure. In denovo diabetic patients, 8 out of 13 patients $(62 \%)$ were found to have hypertension compared to nondiabetic twenty-one $(47 \%)$ patients. The most common type of stroke in all the three cohorts was found to be of the ischemic type with 44, 12 and 30 patients diagnosed with ischemic stroke in diabetic, denovo diabetic and nondiabetic groups respectively.

mRS score outcomes in diabetic, denovo diabetic and nondiabetic stroke patients

The mean mRS score at the time of admission was found to be highest in denovo diabetic stroke patients with a mean score of $4 \pm 0.81$, which did improve significantly with suitable therapeutic interventions and stood at $3.7 \pm 0.85$ at the time of discharge of these patients.

Table 1: Patient demographical, social and type of stroke characteristics in three cohort groups

\begin{tabular}{llll}
\hline & Diabetic (cohort 1) N = 45 & De novo-diabetics (cohort 2) N = 13 & Non-diabetics (cohort 3) N = 45 \\
\hline Age & & & \\
Mean (SD) & $57.8(11.13)$ & $56.69(15.71)$ & $52.54(14.64)$ \\
Median (Range) & $60(40-85)$ & $60(30-85)$ & $51(22-90)$ \\
Male, n(\%) & $32(71)$ & $9(69)$ & $25(56)$ \\
Female, n(\%) & $13(29)$ & $4(31)$ & $20(44)$ \\
Hypertension, n(\%) & $38(84)$ & $8(62)$ & $21(47)$ \\
Alcoholic, n(\%) & $17(38)$ & $8(62)$ & $18(40)$ \\
Smoker, n(\%) & $11(24)$ & $7(54)$ & $11(24)$ \\
Type of stroke: n (\%) & & $12(92)$ & $30(67)$ \\
Ischemic & $44(98)$ & $1(8)$ & $10(22)$ \\
Hemorrhagic & $1(2)$ & & \\
\hline
\end{tabular}

SD: Standard deviation.

Table 2: Mean difference of mRS score in each cohort from time off in hospital admission to discharge

\begin{tabular}{llll}
\hline & mRS score at the time of admission & mRS score at the time of discharge & Mean difference of mRS score \\
\cline { 2 - 4 } & mean \pm SD & mean \pm SD & $0.73 \pm 0.8$ \\
\hline Diabetics & $3.6 \pm 0.81$ & $2.8 \pm 0.95$ & $0.3 \pm 0.63$ \\
Denovo Diabetics & $4 \pm 0.81$ & $3.7 \pm 0.85$ & $0.38 \pm 0.61$ \\
Non-Diabetics & $3.3 \pm 0.78$ & $2.9 \pm 0.83$ & \\
\hline
\end{tabular}

SD: Standard deviation, mRS: modified rankin scale, The mean difference in mRS score was found to be significant between diabetic and nondiabetic stroke patients with a mean difference of $0.73 \pm 0.8(\mathrm{p}<0.0001)$.

Table 3: Comparison of mean difference of mRS score in each cohort by student t-test

\begin{tabular}{lll}
\hline & mean \pm SD & \multicolumn{1}{c}{$\boldsymbol{P}$-value } \\
\hline Diabetics vs Non-Diabetics & $0.73 \pm 0.8$ & $<0.001$ \\
Denovo Diabetics vs Non-Diabetics & $0.30 \pm 0.63$ & 0.1 \\
Denovo Diabetics vs Diabetics & $0.38 \pm 0.61$ & 0.1 \\
\hline
\end{tabular}

SD: Standard deviation, mean \pm SD. All $p$ values are two-sided 


\section{DISCUSSION}

Stroke is a major cause of disability and death especially among geriatrics worldwide. Hyperglycemia is considered as an independent risk factor for stroke. Elevated blood glucose levels worsen the outcomes in acute ischemic stroke patients. Pre-diabetic range blood glucose levels are associated with increased chances of diabetes mellitus, with almost $2 / 3$ rd of the patients progressing to type 2 DM [16]

According to Fonville et al., approximately $1 / 3$ rd of the patients with acute and transient stroke attack with no prior history of diabetes have prediabetes and over $1 / 4$ have diabetes [17]. Recent studies have concluded that patients with pre-diabetic and diabetic range blood glucose levels have poor short-come outcomes in acute stroke patients $[14,18]$.

The main scope of this study was to evaluate the impact of blood glucose levels on outcomes in stroke patients measured by mRS scale.

In our study, there was a male predominance (table 1), with hypertension being most prevalent among diabetic stroke patients. More than half of the patients who were diagnosed as denovo diabetics were alcoholics and smokers.

In our study, we administer mRS scale at the time of in-hospital admission and observed blood glucose levels in enrolled stroke patients. mRS score at in-hospital admission was found to be highest in denovo diabetic patients $4 \pm 0.81$, followed by diabetic stroke patients $3.6 \pm 0.81$ and lastly nondiabetic stroke patients $3.3 \pm 0.78$ (table 2). The patients were clinically managed with appropriate therapeutic interventions to minimise the consequences arising out of stoke along with proper rehabilitation therapy. At the time of discharge, mRS scoring was done. It was observed that diabetic and nondiabetic stroke patients have mRS score of $2.8 \pm 0.95$ and $2.9 \pm 0.83$ respectively (poor outcome is defined as mRS scoze 3 ) compared to $\mathrm{mRS}$ score of $3.7 \pm 0.85$ in denovo diabetic stroke patients at the time of discharge.

The exact mechanism how elevated glucose levels worsens the outcomes in stroke patients in not clearly understood. The proposed mechanisms explaining hypothesis of an impact of hyperglycemia on stroke includes increased acidosis at brain injury site,[19] increased oxidative stress causing further damage at brain injury site,[20] impaired thrombolysis, disturbing recanalisation and causing reperfusion injury [21].

We compared mean difference in mRS score in three cohorts (table 3 ) with student t-test and found a significant difference in outcomes between diabetics and nondiabetics stroke patients of $0.73 \pm 0.8$ $(p<0.001)$. The mean difference in mRS scores of denovo diabetic patients with nondiabetics and diabetics patients was found to be $0.30 \pm 0.63$ and $0.38 \pm 0.61$ respectively with the $p$ value of 0.1 , which is not much significant. This may be attributed to small sample size of denovo diabetic patients recruited in this study, as it was only a 6 mo single center study.

Stress hyperglycemia is common in acute ischemic stroke patients who are previously nondiabetic which should be taken into account, a sudden change in glucose concentrations can adversely impact brain functions and can have a 3 fold high risk in short-term mortality compared to nonhyperglycemic patients [22]. Blood glucose concentrations in hyperglycemic patients in our study were tightly controlled with Insulin therapy in our study, with a target threshold of $<140 \mathrm{mg} / \mathrm{dl}$.

Patients with denovo diabetes in our study had very poor improvement of their mRS score at the time of discharge, even with tighter glycemic control, which suggests in-admission hyperglycemia is a major factor for poor prognosis. It is desirous to rapidly manage glucose levels to achieve normal glucose levels which can reduce overall risk associated with stroke and mortality.

This study had certain limitations. Firstly, our findings are from a small sample and selected part of the patient population which should be interpreted carefully. Moreover it was only a 6 mo study, further follow-up of patients is needed for increased understating on long-term outcomes. Our study was conducted in a single centered resource-limited setting. HbA1c profiles were not conducted to know the blood glucose status in nondiabetic patients.

\section{CONCLUSION}

In conclusion, from this study, we are of opinion that denovo increased blood glucose levels in stroke patients adversely impact the outcomes in these patients, which should be tightly controlled to risks and mortality associated with stroke. Further studies with a larger sample and long-term outcomes measurements would be desirous to establish a definite relation of in hospital admission hyperglycemia and stroke short and long term outcomes.

\section{AUTHORS' CONTRIBUTION}

All authors contributed equally. All authors read and approved the final manuscript

\section{CONFLICTS OF INTERESTS}

Declared none

\section{REFERENCES}

1. Siddiqui AH, Natarajan SK, Hopkins LN, Levy EI. Carotid artery stenting for primary and secondary stroke prevention. World Neurosurg 2011;76:S40-59.

2. WHO. MONICA Project Investigators. The World Health Organization MONICA Project (Monitoring trends and determinants in cardiovascular disease). J Clin Epidemiol 1988;41:105-14.

3. Mukherjee D, Patil CG. Epidemiology and Global burden of stroke. World Neurosurg 2011;76:S85-90.

4. Mackay J, Mensah AG. The Atlas of Heart Disease and Stroke, Deaths from a stroke; WHO-CDC. Available from: http://www.who.int/cardiovascular_diseases/resources/atlas /en/] [Last accessed on 10 Mar 2015]

5. Mozaffarian D, Benjamin EJ, Go AS, Arnett DK, Blaha MJ, Cushman M, et al. Heart disease and stroke statistics-2015 Update. A report from the american heart association. Circulation 2015;131:e29-e322.

6. Pandian JD, Sudhan P. Stroke epidemiology and stroke care services in India. J Stroke 2013;15:128-34.

7. Nagaraja D, Gururaj G, Girish N, Panda S, Roy AK, Sarma GRK, et al. Feasibility study of stroke surveillance: data from Bangalore, India. Indian J Med Res 2009;130:396-403.

8. Karatepe AG, Gunaydin R, Kaya T, Turkmen G. Comorbidity in patients after stroke: impact on functional outcome. J Rehabil Med 2008;40:831-5.

9. O’Donnell MJ, Xavier D, Liu L, Zhang H, Chin SL, Rao-Melacini P, et al. Risk factors for ischemic and intracerebral haemorrhagic stroke in 22 countries (the INTER STROKE study): a casecontrol study. Lancet 2010;376:112-23.

10. Jia Q, Zhao X, Wang C, Wang Y, Yan Y, Li H, et al. Diabetes and poor outcomes within 6 mo after acute ischemic stroke: the China National Stroke Registry. Stroke 2011;42:2758-62.

11. Poppe AY, Majumdar SR, Jeerakathil T, Ghali W, Buchan AM, Hill MD. Admission hyperglycemia predicts a worse outcome in stroke patients treated with intravenous thrombolysis. Diabetes Care 2009;32:617-22.

12. Diener HC, Lees KR, Lyden P, Grotta J, Davalos A, Davis SM, et al. NXY-059 for the treatment of acute stroke: pooled analysis of the SAINT I and II trials. Stroke 2008;39:1751-8.

13. Stead LG, Gilmore RM, Bellolio MF, Mishra S, Bhagra A, Vaidyanathan L, et al. Hyperglycemia as an independent predictor of worse outcome in non-diabetic patients presenting with acute ischemic stroke. Neurocrit Care 2009;10:181-6.

14. Tanaka R, Ueno $\mathrm{Y}$, Miyamoto N, Yamashiro K, Tanaka Y, Shimura $\mathrm{H}$, et al. Impact of diabetes and prediabetes on the short term prognosis in patients with acute ischemic stroke. J Neurol Sci 2013;332:45-50.

15. Jia Q, Liu G, Zheng $\mathrm{H}$, Zhao X, Wang C, Wang $\mathrm{Y}$, et al. Impaired glucose regulation predicted $1 \mathrm{y}$ mortality of chinese patients with ischemic stroke: data from abnormal glucose regulation in patients with an acute stroke across China. Stroke 2014;45:1498-500. 
16. Buysschaert M, Bergman M. Definition of pre diabetes. Med Clin North Am 2011;95:289-97.

17. Fonville S, Zandbergen AA, Vermeer SE, Dippel DW, Koudstaal PJ, den Hertog HM, et al. Prevalence of prediabetes and newly diagnosed diabetes in patients with a transient ischemic attack or stroke. Cerebrovasc Dis 2013;36:283-9.

18. Osei E, Fonville S, Zandbergen AA, Koudstaal PJ, Dippel DW, den Hertog HM. Glucose in prediabetic and diabetic range and outcome after stroke. Acta Neurol Scand 2017;135:170-5.

19. Siemkowicz E, Hansen AJ. Brain extracellular ion composition and EEG activity following $10 \mathrm{~min}$ ischemia in normo-and hyperglycaemic rats. Stroke 1981;12:236-40.

20. Robbins NM, Swanson RA. Opposing effects of glucose on stroke and reperfusion injury: acidosis, oxidative stress, and energy metabolism. Stroke 2014;45:1881-6.
21. Luitse MJ, Biessels GJ, Rutten GE, Kappelle LJ. Diabetes, hyperglycaemia, and acute ischaemic stroke. Lancet Neurol 2012;11:261-71.

22. Capes SE, Hunt D, Malmberg K, Pathak P, Gerstein HC. Stress hyperglycemia and prognosis of stroke in nondiabetic and diabetic patients: a systematic overview. Stroke 2001; 32:2426-32.

\section{How to cite this article}

- $\quad$ Syed Junaid Ahmed, Abdur Rahman Mohd Masood, Safiya Sumana, Khadeer Ahmed Ghori, Javed Akhtar Ansari, Musa Khan. Stroke outcomes in nondiabetic, denovo diabetic and diabetic Indian patients measured by modified rankin scale: an observational study. Int J Pharm Pharm Sci 2017;9(8):94-97. 